\title{
Horizontal and Declined Squats in Healthy Individuals: A Study of Kinematic and Muscle Patterns
}

\author{
Valdeci Carlos Dionisio, ${ }^{1}$ Bruna Maria Silva Azevedo, ${ }^{2}$ and Daniela Abrahão Siqueira ${ }^{2}$ \\ ${ }^{1}$ Physical Therapy Course, Federal University of Uberlândia, Benjamin Constant Street No. 1286, 38400-678 Uberlândia, MG, Brazil \\ ${ }^{2}$ Physical Therapy Course of the University Center of Triângulo, Nicomedes Alves dos Santos Avenue No. 4545, \\ 38411-106 Uberlândia, MG, Brazil
}

Correspondence should be addressed to Valdeci Carlos Dionisio; vcdionisio@gmail.com

Received 30 October 2013; Accepted 5 December 2013

Academic Editors: K.-H. Lin and A. Ozcan Edeer

Copyright (c) 2013 Valdeci Carlos Dionisio et al. This is an open access article distributed under the Creative Commons Attribution License, which permits unrestricted use, distribution, and reproduction in any medium, provided the original work is properly cited.

\begin{abstract}
Squats are frequently incorporated in physiotherapy programmes and performed in different ways. Accordingly, muscle and kinematic patterns also differ. The objective was to compare the kinematics and EMG patterns of the major muscles of knee and ankle joints during different squat exercises on horizontal (HP) and $25^{\circ}$ decline (DP) boards. Seventeen healthy individuals performed squats on HP and DP with bilateral support and restriction of trunk movements in the sagittal plane. The knee and ankle angular displacements in the sagittal plane and the EMG activities of the major muscles of the lower limb in four subphases of movement were recorded and analyzed. For the descending phase of the HP squats, the angular excursion, as well as the initial and final positions, was smaller for the knee $(P<0.05)$ but larger for the ankle $(P=0.01)$. For the ascending phase of the HP squats, the initial and final positions were larger for the ankle $(P<0.02)$ and the final position was smaller for the knee $(P=0.05)$. All muscles remained activated and showed similarity between the tasks $(P>0.44)$, except for the tibialis anterior in both squat exercises $(P<0.03)$. The HP and DP squats produced different kinematics (knee and ankle joints) but did not modify the EMG strategy for both movement phases.
\end{abstract}

\section{Introduction}

Injuries of the knee joint account for numerous lesions that affect the lower extremities [1]. In any lesion involving the knee, a reflex inhibition of the quadriceps is observed, accompanied by hypotrophy $[2,3]$, which causes knee instability [4]. This instability contributes to chronic pain, increasing the inhibition process and weakening the quadriceps and, in turn, leading to dysfunctions of the extensor mechanism [5]. Under these circumstances, quadriceps strengthening is essential to stabilize and minimize or reverse the process of inhibition and muscle weakening; such strengthening is therefore the main challenge that confronts the rehabilitation process [4].

Several protocols have been suggested for recovering quadriceps force, but multijoint exercises are the preferred form of therapy for dysfunctions of the extensor mechanism $[6,7]$. Multijoint exercises are regarded as advantageous because they simulate functional activities $[6,8]$, exert minimal stress on the patellofemoral joint during the functional range of movements [6], and enable muscle cocontraction, which contributes to joint stability [9]. An effective multijoint exercise extensively used in clinical practice by physiotherapists is the squat $[7,10]$. Some studies have demonstrated the usefulness of performing squats during knee rehabilitation $[7,10]$ - an effectiveness that may be attributed to the fact that such exercises increase the muscle activities of the hip, knee joints, and ankle joints; this increase is supported by the high electromyographic (EMG) activities of the hamstrings, quadriceps, and gastrocnemius muscles [11]. Some researchers have suggested that squats performed on a $25^{\circ}$ decline plane (DP) more effectively treat patellar tendinitis than do traditional squats performed on a horizontal plane (HP) [12-14]. The DP squat is performed on a platform sufficiently large to enable positioning the feet at an incline $25^{\circ}$ to the surface (heel elevation). The tibia is positioned 
vertically and strong plantar flexion of the ankle is observed. The literature discusses several indications that quadriceps muscles produce more force in DP than in HP given greater quadriceps EMG activity [13-15], greater effort (tension) of the patellar tendon [15-17], and greater knee joint torque $[14,17]$. In all these studies [14-17], however, squatting tasks were performed with unipodal (single leg) support and without any type of trunk control (displacement in the sagittal plane). Trunk position and bipodal (both legs) support can modify the position of the centre of mass (COM), as well as the pattern and magnitude of EMG activity of the major muscles of the lower limb, thereby enabling individuals to maintain balance and perform tasks [18]. During the initial phase of a rehabilitation programme, multijoint exercises are performed with bipodal support and, sometimes, with control over the trunk position (e.g., trunk upright). DP and $\mathrm{HP}$ squats have not been tested under these conditions, and the related kinematics and EMG patterns remain uncertain. If EMG activities are higher under DP squats than under HP squats (similar to that observed in previous studies, in which exercises were performed under unipodal support), using DP squats would be the therapy of preference for recovering quadriceps force across all the phases of a rehabilitation programme.

Clarifying this issue is an important consideration for coaches and physiotherapists, especially those focusing on rehabilitating quadriceps force. The present study aimed to compare the kinematics and EMG patterns of the major muscles of knee and ankle joints during the descending and ascending phases of HP and DP squats. Bipodal support and restriction of trunk movements in the sagittal plane were applied.

\section{Methods}

Seventeen healthy individuals ( 8 men, 9 women) aged 1835 years participated in the study. The means and standard deviations of age, weight, and height were $22.8 \pm 3.14$ years, $65.4 \pm 13.18 \mathrm{~kg}$, and $169 \pm 0.08 \mathrm{~cm}$, respectively. All the individuals are right-handed and weekend athletes who do not exercise regularly. The exclusion criteria were a history of pain that suggests joint or muscle disorder and surgery or musculoskeletal disorders in the lower limbs, spine, or shoulders. The individuals signed an informed consent form approved by the Research Ethics Committee (protocol 634853/2007).

After shaving and skin asepsis with alcohol, active bipolar surface electrodes (DataHominis Tecnologia Ltda) were positioned on the vastus medialis obliquus (VMO), vastus lateralis (VL), biceps femoris (BF), tibialis anterior (TA), soleus (SO), and erector spinae (EE) muscles. For the VMO, surface electrode was placed on the belly muscle along the direction of the fibres (about $54^{\circ}$ ). To identify the motor point, the participants were asked to perform an isometric contraction of the quadriceps so that the VMO activity could be observed [19]. Then, an electrode was placed $2 \mathrm{~cm}$ distal from the motor point. For the other muscles, electrodes were placed in accordance with the guidelines of the Surface Electromyography for the Noninvasive Assessment of Muscles project of the European Union's Biomedical Health and Research Program (BIOMED II) [20]. Each electrode consists of two parallel silver plates measuring $1 \mathrm{~cm}$ long and $1 \mathrm{~mm}$ wide, positioned $1 \mathrm{~cm}$ apart. The electrodes had a gain of 20 (amplification), an impedance of $10 \mathrm{G} \Omega$, and a common mode rejection ratio of $84 \mathrm{~dB}$. They were connected to computer-aided EMG equipment (DataHominis Tecnologia Ltda) capable of 100time amplification (2000-time total amplification). It had a $15 \mathrm{~Hz}$ to $1 \mathrm{kHz}$ bandpass filter and an acquisition frequency of $2000 \mathrm{~Hz}$. A $3 \mathrm{~cm}^{2}$ ground electrode was also attached to the right iliac crest and daubed with electroconductive gel to improve signal transfer. An auxiliary channel was also used with a DataHominis electrogoniometer with flexible poles and $360^{\circ}$ rotation. It was placed on the knee and ankle joints (lateral epicondylus femoris and $2 \mathrm{~cm}$ below the lateral malleolus). Before the assessment, the electrogoniometer channels were calibrated to determine the maximum range (i.e., $180^{\circ}$ ). For total knee extension, this range was $0^{\circ}$ and for flexion, the range was any value greater than $0^{\circ}$. The maximum range for the ankle was the same $\left(0^{\circ}\right.$ to $\left.180^{\circ}\right)$, but the initial position was estimated at around $90^{\circ}$. A range greater than $90^{\circ}$ indicates dorsiflexion, whereas a range less than $90^{\circ}$ reflects plantar flexion.

The individuals performed HP and DP squats, both in the ascending and descending phases separately. They were positioned with feet parallel and shoulder-width apart, but the angle of the feet relative to the boards was chosen according to individual preference. In general, this angle varied from $5^{\circ}$ to $8^{\circ}$ of external rotation. The participants were also advised to keep their arms flexed at $90^{\circ}$ in relation to the shoulders and use it as a single rigid body (without moving the elbow, wrist, and hand) to guide movement (Figure 1). This position was considered partly restrictive of trunk movements in the sagittal plane (trunk upright), thereby allowing for the same strategy in performing the squats; in previous studies, this position was also adopted for HP squats $[19,21,22]$ and sit-to-stand exercises [22]. The EMG signals were recorded only from the right-hand side. The electrical activity of the muscles was captured during horizontal and $25^{\circ}$ decline squats in both descending and ascending phases. The ranges of motion for the descending and ascending phases were $0^{\circ}$ to $70^{\circ}$ and $70^{\circ}$ to $0^{\circ}$ of knee flexion, respectively. For the decline squats, a platform declined at an angle of $25^{\circ}$ was used for the ankle positioned in plantar flexion. An adjustable support was placed behind the participants to restrain the final movement of the descending phase and to mark the onset of the ascending phase movement. The support served as a guide, but the participants were prohibited from resting on support (Figure 1).

Each individual was allowed two repetitions for him/her to learn the movements and to verify whether any electrode wire limited his/her movements. The individuals performed 10 repetitions of the HP squats, with five of the repetitions executed in the ascending phase and five in the ascending phase. Then, they performed 10 repetitions of the DP squats, 

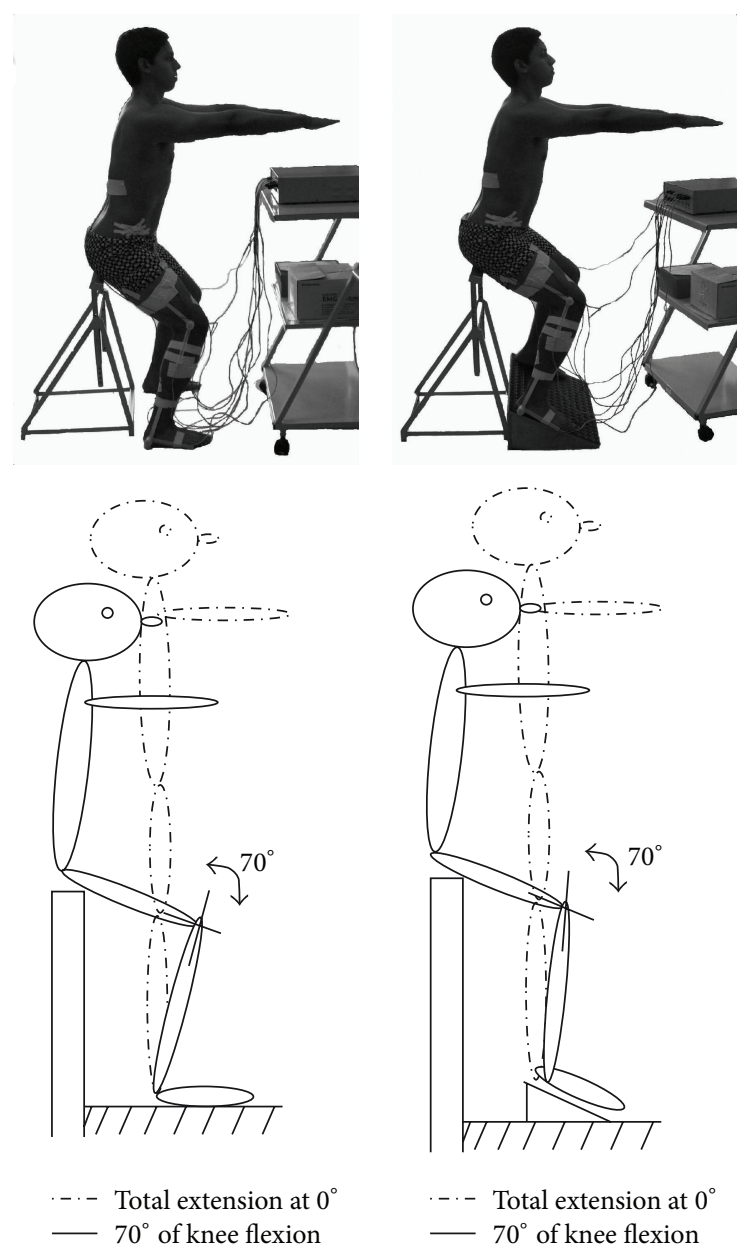

(a)

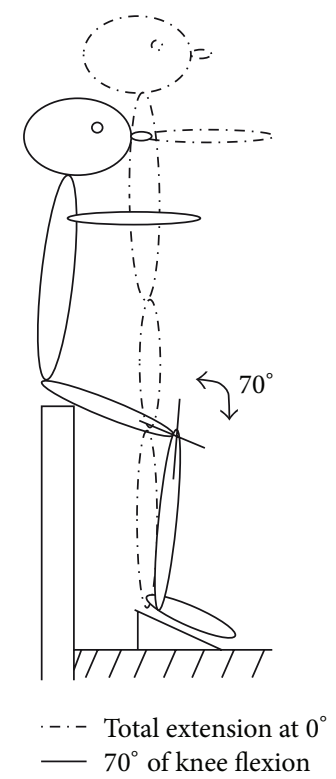

(b)

FIGURE 1: Task model for squats performed in HP (a) and DP (b). During the descending phase, movement ranged from $0^{\circ}$ to $70^{\circ}$ of the knee flexion, and, during the ascending phase, the movement ranged from $70^{\circ}$ to $0^{\circ}$ of the knee flexion.

with five of the repetitions executed in the ascending phase and five in the ascending phase. Between each set of five repetitions, an interval of 1 minute was allocated. For each squat (HP and DP), the participants were given a 3-minute rest period to avoid muscle fatigue.

A verbal instruction from the researcher signalled the onset of each movement. The individuals were instructed to perform the movements as quickly as possible and to maintain the final position (FP) until the end of data collection, which lasted 2 seconds.

Electrogoniometric signals were obtained in degrees, filtered at $50 \mathrm{~Hz}$, and calculated as the velocities of the knee and ankle joints. The EMG signals, obtained in microvolt $(\mu \mathrm{V})$, were rectified (full wave), filtered at $25 \mathrm{~Hz}$, and normalized by maximal voluntary isometric contraction (MVIC). For all MVIC tests, the participants were seated in a comfortable chair [19]. The MVICs of all portions of the quadriceps were tested with the knee of the participant fixed manually at $20^{\circ}$ of flexion ( $0^{\circ}$ equal to full extension), and resistance was applied to the tibia just above the ankle in the flexion direction. The MVIC of the biceps femoris was tested with the knee of the participant fixed manually at $90^{\circ}$, and resistance was applied to the tibia just above the ankle in the extension direction. For the MVIC of the tibialis anterior, the knee was at full extension and the ankle was fixed manually at $90^{\circ}$; resistance was applied to the forefoot in the plantar flexion direction. The MVIC of the soleus was tested with the knee flexed at $90^{\circ}$ and the foot positioned on the ground; resistance was applied over the knee in the ground direction to avoid knee movements in the superior direction. Finally, for MVIC of the erector spinae, the knees were flexed at $40^{\circ}$, the feet were flat on the ground, the arms were positioned over the chest, and the trunk was fixed manually on the scapulas [23]; resistance was applied in the anterior direction. On the basis of knee velocity, the EMG signals were analyzed from four subphases of movement: (1) subphase 1,100 ms just before the knee velocity first achieved $5 \%$ of its peak (the beginning of movement); (2) subphase 2, defined as the acceleration time of the squat, that is, the interval between the end of subphase 1 and the time at which knee velocity achieved its peak; (3) subphase 3, defined as the deceleration time of the squat, and the interval between the end of subphase 2 and the time at which knee velocity returned to $5 \%$ of its peak; and (4) subphase 4, defined as the time point during which the body remained at the target squat position for $100 \mathrm{~ms}$ following the end of subphase 3 . The mean of the five repetitions of each individual for each of the phases (ascending and descending) of the tasks (HP and DP squats) was obtained. The integral of each muscle for each subphase of movement was calculated on the basis of the mean of each individual. These procedures were carried out in Excel (Microsoft Office XP 2003) and KaleidaGraph (Synergy, version 3.08).

To evaluate the kinematic variables (angular displacement and maximal velocity of the knee and ankle joints), we employed the $t$-test for dependent samples. To evaluate the effect of the tasks (HP and DP squats), we used twoway factorial ANOVA for repeated measures (task (HP and DP) $\times$ muscle (curve integral of each muscle separately)) As a post hoc test, we used Tukey's honestly significant difference method to test significant differences between specific subphases. The data were analyzed in a personal computer using Statistica for Windows (Statsoft, Inc., version 5.0). For all evaluations, the significance level $(\alpha)$ was set at 0.05 , with $90 \%$ power.

\section{Results}

For the descending phase of the movements, the $t$-test revealed that during the DP squats, the ankle exhibited a larger plantar flexion in the initial position (IP) and a lower range in the FP and angular excursion (AE) than during the $\mathrm{HP}$ squats. For the knee, the DP squats produced a larger knee flexion in IP, FP, and AE than did the HP squats (Table 1). Moreover, maximal ankle velocity was greater during the HP squats than during the DP squats. The maximal knee velocity was similar in both squatting movements (Table 1). 
TABLE 1: Kinematic variables across all subjects for phases of the squats.

\begin{tabular}{|c|c|c|c|c|c|c|c|c|}
\hline \multirow{2}{*}{ Variable $(\mathrm{dg})^{\mathrm{a}}$} & \multicolumn{4}{|c|}{ Descent phase } & \multicolumn{4}{|c|}{ Ascent phase } \\
\hline & $\mathrm{HP}^{\mathrm{b}}$ mean $(\mathrm{SD})^{\mathrm{d}}$ & $\mathrm{DP}^{\mathrm{c}}$ mean $(\mathrm{SD})$ & $t$ value & $P$ value & HP mean (SD) & DP mean (SD) & $t$ value & $P$ value \\
\hline Knee IP & $2.30(5.13)$ & $3.84(5.23)$ & -2.15 & $0.04^{*}$ & $65.00(10.19)$ & $67.32(11.06)$ & -1.61 & 0.12 \\
\hline Knee $\mathrm{FP}^{\mathrm{f}}$ & $61.89(9.97)$ & $67.82(10.15)$ & -4.86 & $0.01^{*}$ & $2.25(5.41)$ & $5.12(6.61)$ & -2.08 & $0.05^{*}$ \\
\hline Knee $\mathrm{AE}^{\mathrm{g}}$ & $59.58(10.77)$ & $63.98(10.86)$ & -3.54 & $0.01^{*}$ & $62.74(10.57)$ & $62.19(10.71)$ & 0.41 & 0.68 \\
\hline Ankle IP & $85.38(5.59)$ & $75.42(5.21)$ & 9.68 & $0.01^{*}$ & $94.98(8.24)$ & $89.28(5.95)$ & 3.22 & $0.01^{*}$ \\
\hline Ankle FP & $101.75(6.58)$ & $89.40(6.56)$ & 12.65 & $0.01^{*}$ & $79.71(7.78)$ & $75.57(7.69)$ & 2.58 & $0.02^{*}$ \\
\hline Ankle AE & $16.37(3.23)$ & $13.97(5.13)$ & 3.26 & $0.01^{*}$ & $15.26(4.61)$ & $13.71(5.10)$ & 1.93 & 0.07 \\
\hline Maximal knee velocity $(\mathrm{dg} / \mathrm{s})^{\mathrm{h}}$ & $295.63(89.93)$ & $283.94(78.93)$ & 0.59 & 0.55 & $62.74(53.37)$ & $54.00(54.67)$ & 0.73 & 0.47 \\
\hline Maximal ankle velocity (dg/s) & $75.04(20.24)$ & $58.84(24.20)$ & 4.60 & $0.01^{*}$ & $15.03(7.9)$ & $12.67(9.82)$ & 1.07 & 0.29 \\
\hline
\end{tabular}

${ }^{\mathrm{a}}$ Degrees: dg; ${ }^{\mathrm{b}}$ horizontal plane: HP; ${ }^{\mathrm{c}}$ decline plane: DP; ${ }^{\mathrm{d}}$ standard deviation: SD; ${ }^{\mathrm{e}}$ initial position: IP (measured in degrees); ${ }^{\mathrm{f}}$ final position: FP; ${ }^{\mathrm{g}}$ angular excursion: AE; ${ }^{\text {h }}$ degrees by second $(\mathrm{dg} / \mathrm{s}) ;{ }^{*}$ statistical significance.

For the ascending phase of the movements, the $t$-test revealed no difference between the squats for the IP and AE of the knee joint. The FP of this joint exhibited a difference between squats. The IP and FP of the ankle joint also exhibited a difference, but the AE was similar between squats (Table 1). With regard to the maximal knee and ankle velocities, no difference was observed between the squatting movements (Table 1).

For the descending phase of the movements (Figure 2), ANOVA revealed no effect of the squats on EMG activity $(P>0.44)$, except for TA $(P=0.03)$. However, ANOVA revealed a difference in the subphases of movement for all the muscles $(P<0.01)$; interaction between the squats and subphases was observed only in TA $(P=0.02)$. The post hoc analysis showed that this difference occurred in subphase 3 (deceleration), with higher activity in the HP squats than in the DP squats $(P<0.01)$. The other subphases were similar between squats $(P>0.91)$.

For the ascending phase of the movements (Figure 3), ANOVA again revealed no effect of the squats on EMG activity $(P>0.46)$, but differences were found in all the muscles with regard to the subphases $(P<0.01)$; interaction between the squats and subphases was found only in TA $(P=0.02)$. The post hoc analysis revealed that this difference occurred during subphase 1, with higher activity in the HP squats than in the DP squats $(P<0.02)$. The other subphases were similar between squats $(P>0.75)$.

\section{Discussion}

The primary aim of the study was to evaluate the kinematics and EMG patterns of the main muscles that produce movements in the knee and ankle joints during bipodal squats in HP and DP, with restriction of trunk movements in the sagittal plane. The control of trunk movements in the sagittal plane was reported in previous studies, showing that different individuals can perform squats $[19,21]$ or sit-tostand exercises [22] in a similar manner through repetitions. The performance of the participants revealed the use of a common strategy, in which the knee and ankle joints direct movement and exhibit large joint torques.
During the descent phase of the movements, differences in kinematic variables were found (Table 1). The strong plantar flexion in the DP squats influenced the position of the other joints during the standing position (IP) and the squat position (FP). This influence was reflected in AE. Although some researchers did not find differences in various declination angles-that is, $0^{\circ}, 8^{\circ}, 16^{\circ}$, and $24^{\circ}$ [17] - the limitation imposed on the ankle joint in DP (strong plantar flexion) resulted in a small ankle velocity, compensated for by strong knee flexion. The same results were also derived in previous studies $[15,16]$. Dorsiflexion is greater during HP squats than during $\mathrm{DP}$ squats, thereby producing greater $\mathrm{AE}$ and ankle velocity, which leads to a more horizontal position (anterior displacement) of the tibia $[17,24]$.

Coactivation of the muscles that act on the ankle was reported by many studies on squatting movements [18, 25]. This coactivation was also observed in the present study, specifically between TA and SO muscles, with greater activation during the HP squats than in the DP squats (Figures 2(b) and 2(d)). The more horizontal position of the tibia [24] increased the dorsiflexion of the ankle [17] and the demand on the TA muscles in the HP squats. On the other hand, during the DP squats, the position of the ankle increased the TA length, leading to a length-tension relationship that is inappropriate for good activation [25]. To counter the anterior displacement of the tibia caused by the action of the TA muscles and gravitational force, the SO muscles are activated to decelerate during the descending phase of the squats, aiding the anteroposterior stability of the ankle and maintenance of balance [25].

Despite these differences in kinematics and in TA EMG activity, the quadriceps did not present a difference between tasks. During acceleration (subphase 2), the gravitational force led to knee flexion, and the VMO, VL, and BF muscles (Figures 2(a), 2(c), and 2(e)) maintained low activities [19, 25]. Conversely, during subphase 3 , the quadriceps showed increased EMG activity, being coactivated with the BF muscles (Figures 2(a), 2(c), and 2(e)), as well as with the EE muscles [19] for knee [19, 26] and pelvis stabilization, respectively; this coactivation avoids excessive hip flexion [19, 21] whilst maintaining control over the trunk. The eccentric action of the quadriceps (VMO and VL) was necessary in 


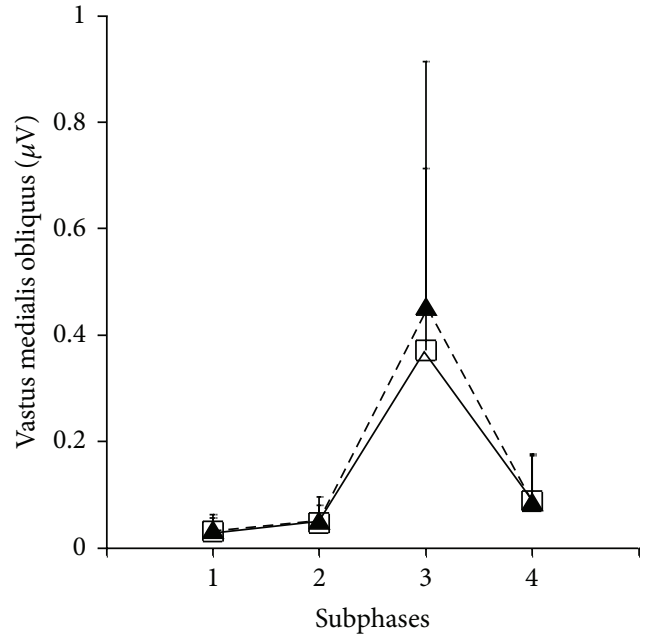

(a)

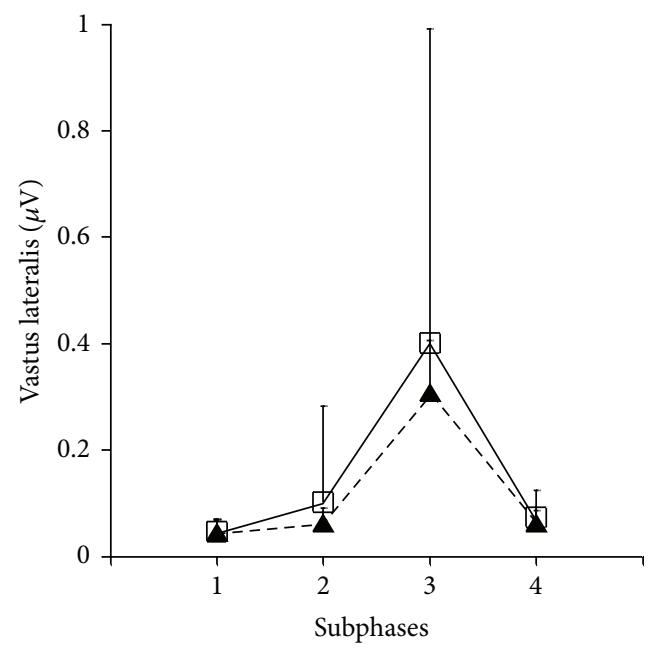

(c)

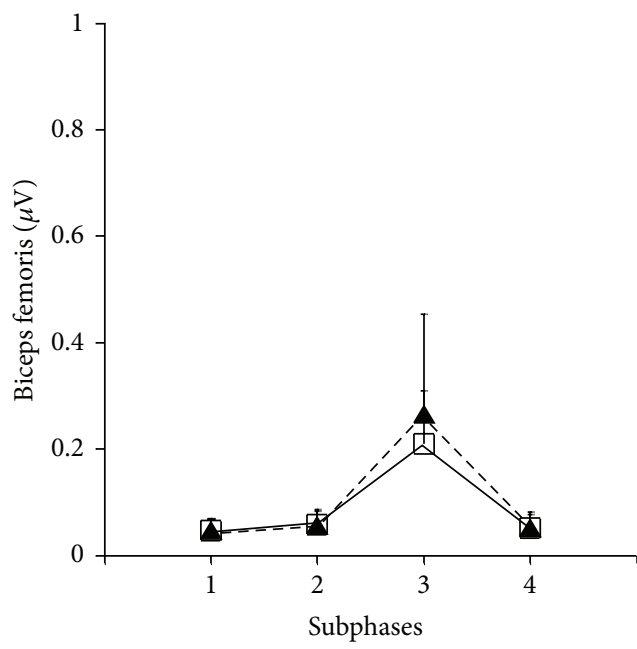

$\square$ Horizontal

-ム- Decline

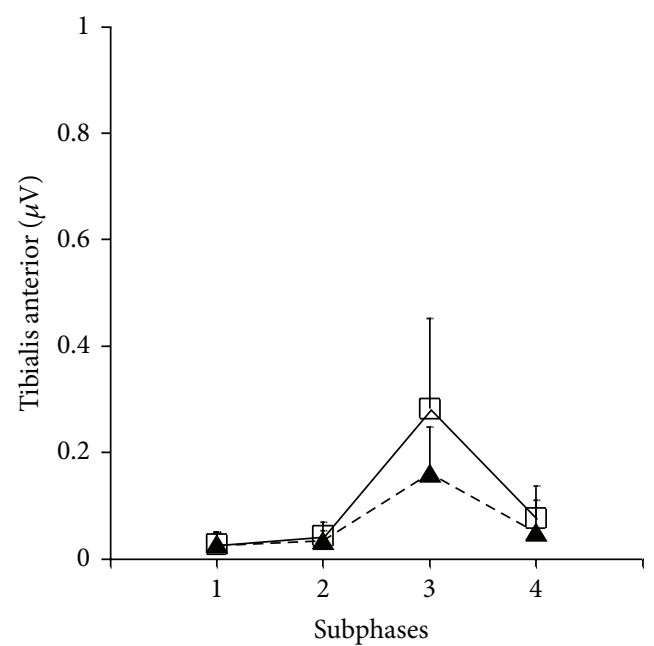

(b)

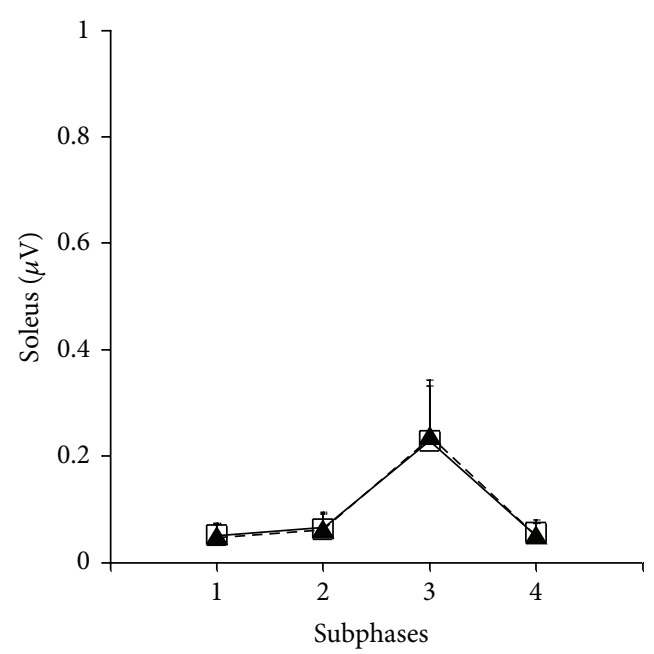

(d)

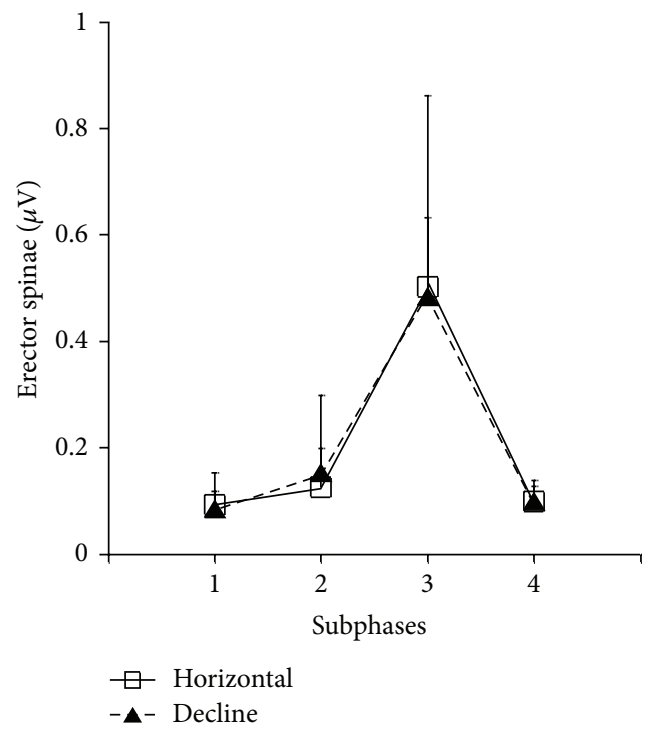

(f)

FIGURE 2: Mean values and standard deviation across all individuals to all muscles (VMO, VL, BF, TA, SO, and EE) during the descending phase for both squats (HP, open squares; DP, closed triangles) in all subphases of the movement. 


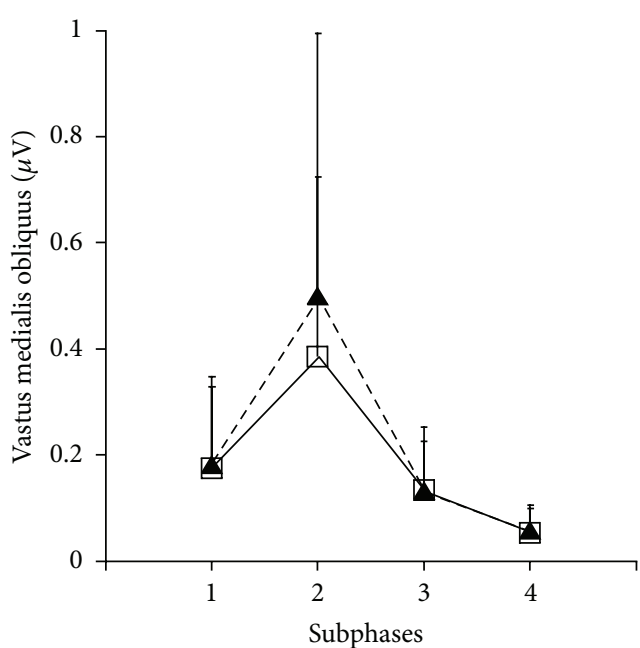

(a)

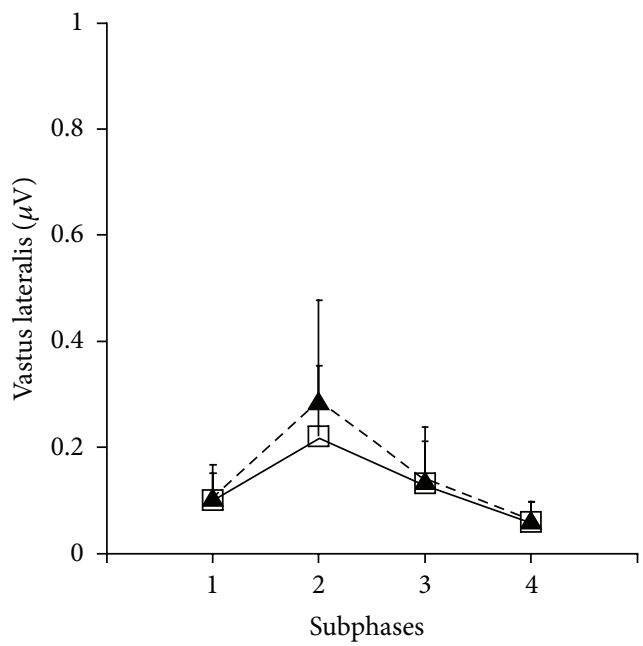

(c)

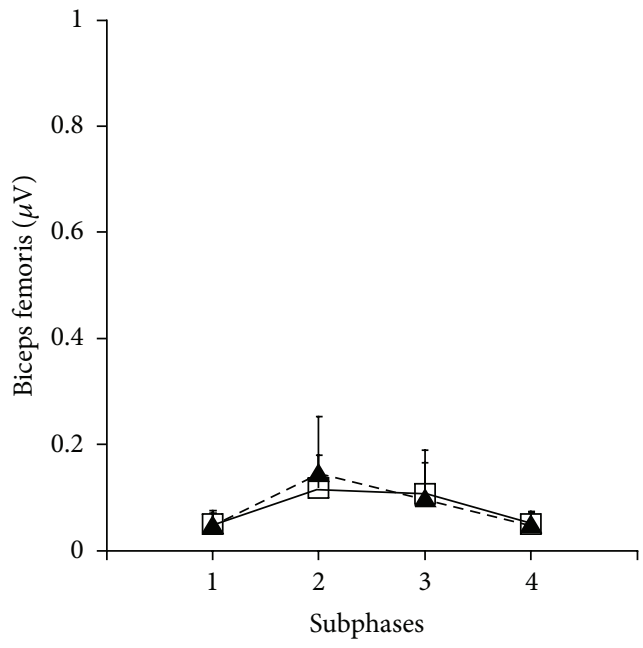

$\square$ Horizontal

- - Decline

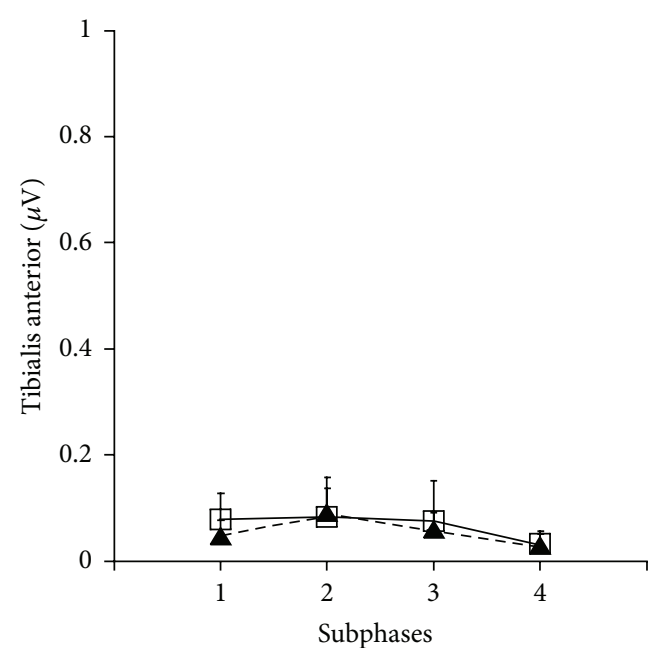

(b)

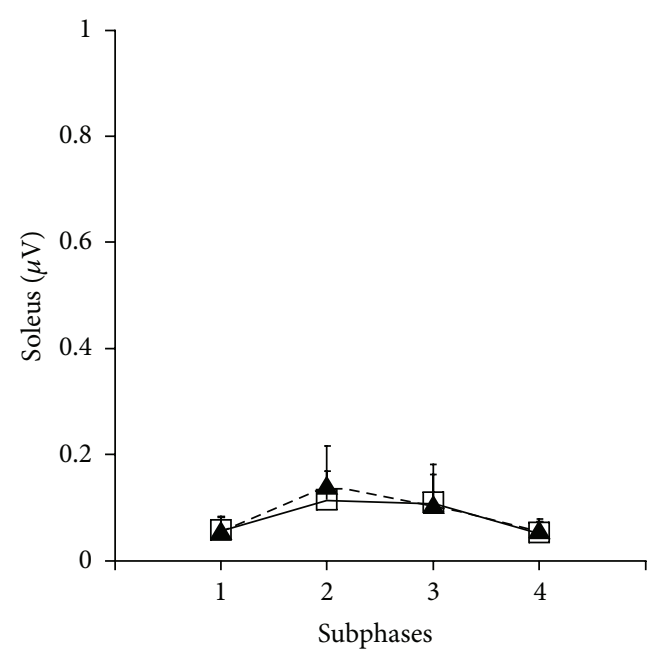

(d)

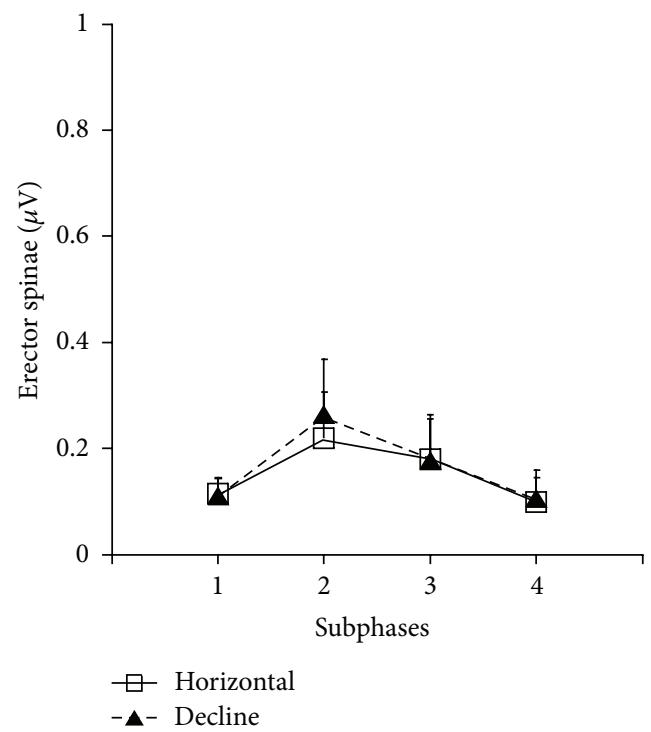

(f)

FIGURE 3: Mean values and standard deviation across all individuals to all muscles (VMO, VL, BF, TA, SO, and EE) during the ascending phase for both squats (HP, open squares; DP, closed triangles) in all subphases of the movement. 
both tasks for decelerating gravitational action $[19,25,26]$ and for identifying the target position ( $70^{\circ}$ flexion). However, some studies demonstrated that DP squats presented greater demand on the extensor knee mechanism than did HP squats, but these studies were carried out with unipodal support. With unipodal support, joint demand is accentuated (gravitational force), which increases the need for a stronger activation of the quadriceps to decelerate movement [25]. This activation can produce up to a $30 \%$ increase in eccentric work, mean and peak patellar tendon force, and angle at peak force relative to those derived under bipodal support [15]. In the current work, similar mechanical demands on the joints involved characterized the similarities in action of the quadriceps muscles between the squat movements.

Another important aspect is the anteroposterior displacement of the trunk, which influences COM displacement [19]. The anterior displacement of the COM favoured the decrease in the EMG activity demand on the quadriceps in the HP squats. In the DP squats, on the other hand, the $\mathrm{COM}$ was further displaced toward the posterior direction, consequently increasing the demand on the quadriceps [14]. This displacement was due to the plantar flexion of the ankle. In previous studies, unipodal support was applied without control over the trunk position and a higher EMG activity of the quadriceps was found $[14,15,17]$. In the present study (with bipodal support), trunk movements in the sagittal plane were controlled, thereby avoiding anterior trunk displacement. This control also explained the similar activation of the quadriceps in both tasks. However, Alves et al. [25] who also studied squats with bipodal support, did not control trunk movements and observed a similarity between tasks. This result suggests that type of support (unipodal or bipodal) is more important than trunk control in influencing the EMG activities under different types of squats (HP and DP). Nevertheless, further studies should be conducted to confirm this hypothesis.

For the ascent phase of the squats, the HP squats presented greater IP and FP of the ankle but lower FP of the knee than did the DP squats. The other variables, including knee and ankle velocities, were not influenced by ankle position. For this phase of the squat, the knee and ankle velocities were small because acceleration depends on muscular activation to overcome inertial forces for the generation of movement [21]. For the TA muscles, however, a difference between squats was found; this difference required greater activity in the HP squats than in the DP squats (Figure 3(b)). It was also influenced by the position of the ankle, which needs more stability given the anterior displacement of the tibia. To overcome inertia and enable the body to perform ascending movements, all the muscles increased their EMG activities in both tasks during acceleration (Figure 3). The VMO and VL muscles were activated to enable knee extension, and the $\mathrm{BF}$ muscles were activated to enable pelvis pushing movements and maintain hip extension [26, 27]. The EE muscles were also activated (Figure 3(f)), thereby aiding the BF muscle in controlling trunk flexion and maintaining its extension [25]. In this ascent phase of the squat, the TA and SO muscles were coactivated in a similar manner between the tasks (Figures 5(b) and 5(d)), which provided ankle stability.
In summary, we showed that DP and HP squats have the same EMG patterns in the quadriceps, indicating that DP squats are not necessarily more effective than HP squats. Therefore, in the early stages of a rehabilitation programme for recovering quadriceps force, both DP and HP squats can be performed under bipodal support. Nonetheless, our results should be interpreted with caution because the movements studied do not fully correspond to the exercises used for knee rehabilitation. In particular, the participants were instructed to execute the moves as quickly as possible; thus, such movements cannot be extrapolated to the recommendations of the American College of Sports Medicine with regard to squats.

\section{Conclusion}

DP and HP squats with bipodal support and control of trunk movements in the sagittal plane produced different kinematics (ankle and knee joints) but did not modify the EMG activities in HP and DP squats, as well as in descending and ascending phases of the movements.

\section{Conflict of Interests}

The authors declare that there is no conflict of interests.

\section{References}

[1] J. Yang, S. W. Marshall, J. M. Bowling, C. W. Runyan, F. O. Mueller, and M. A. Lewis, "Use of discretionary protective equipment and rate of lower extremity injury in high school athletes," American Journal of Epidemiology, vol. 161, no. 6, pp. 511-519, 2005.

[2] Y. Okada, "Histochemical study on the atrophy of the quadriceps femoris muscle caused by knee joint injuries of rats," Hiroshima Journal of Medical Sciences, vol. 38, no. 1, pp. 13-21, 1989.

[3] R. M. Palmieri, J. A. Tom, J. E. Edwards et al., "Arthrogenic muscle response induced by an experimental knee joint effusion is mediated by pre- and post-synaptic spinal mechanisms," Journal of Electromyography and Kinesiology, vol. 14, no. 6, pp. 631-640, 2004.

[4] C. M. Powers, "Rehabilitation of patellofemoral joint disorders: a critical review," Journal of Orthopaedic and Sports Physical Therapy, vol. 28, no. 5, pp. 345-354, 1998.

[5] D. A. Rice and P. J. McNair, "Quadriceps arthrogenic muscle inhibition: neural mechanisms and treatment perspectives," Seminars in Arthritis and Rheumatism, vol. 40, no. 3, pp. 250266, 2010.

[6] L. A. Steinkamp, M. F. Dillingham, M. D. Markel, J. A. Hill, and K. R. Kaufman, "Biomechanical considerations in patellofemoral joint rehabilitation," American Journal of Sports Medicine, vol. 21, no. 3, pp. 438-444, 1993.

[7] S. F. Tang, C. K. Chen, R. Hsu, S. W. Chou, W. H. Hong, and H. L. Lew, "Vastus medialis obliquus and vastus lateralis activity in open and closed kinetic chain exercises in patients with patellofemoral pain syndrome: an electromyographic study," Archives of Physical Medicine and Rehabilitation, vol. 82, no. 10, pp. 1441-1445, 2001. 
[8] J. Nyland, T. Brosky, D. Currier, A. Nitz, and D. Caborn, "Review of the afferent neural system of the knee and its contribution to motor learning," Journal of Orthopaedic and Sports Physical Therapy, vol. 19, no. 1, pp. 2-11, 1994.

[9] J. T. Podraza and S. C. White, "Effect of knee flexion angle on ground reaction forces, knee moments and muscle cocontraction during an impact-like deceleration landing: implications for the non-contact mechanism of ACL injury," Knee, vol. 17, no. 4, pp. 291-295, 2010.

[10] E. Witvrouw, R. Lysens, J. Bellemans, K. Peers, and G. Vanderstraeten, "Open versus closed kinetic chain exercises for patellofemoral pain: a prospective, randomized study," American Journal of Sports Medicine, vol. 28, no. 5, pp. 687-694, 2000.

[11] R. F. Escamilla, "Knee biomechanics of the dynamic squat exercise," Medicine and Science in Sports and Exercise, vol. 33, no. 1, pp. 127-141, 2001.

[12] C. R. Purdam, P. Johnsson, H. Alfredson, R. Lorentzon, J. L. Cook, and K. M. Khan, "A pilot study of the eccentric decline squat in the management of painful chronic patellar tendinopathy," British Journal of Sports Medicine, vol. 38, no. 4, pp. 395-397, 2004.

[13] M. A. Young, J. L. Cook, C. R. Purdam, Z. S. Kiss, and H. Alfredson, "Eccentric decline squat protocol offers superior results at 12 months compared with traditional eccentric protocol for patellar tendinopathy in volleyball players," British Journal of Sports Medicine, vol. 39, no. 2, pp. 102-105, 2005.

[14] M. Kongsgaard, P. Aagaard, S. Roikjaer et al., "Decline eccentric squats increases patellar tendon loading compared to standard eccentric squats," Clinical Biomechanics, vol. 21, no. 7, pp. 748754, 2006.

[15] A. Frohm, K. Halvorsen, and A. Thorstensson, "Patellar tendon load in different types of eccentric squats," Clinical Biomechanics, vol. 22, no. 6, pp. 704-711, 2007.

[16] J. Zwerver, S. W. Bredeweg, and A. L. Hof, "Biomechanical analysis of the single-leg decline squat," British Journal of Sports Medicine, vol. 41, no. 4, pp. 264-268, 2007.

[17] J. Richards, D. Thewlis, J. Selfe, A. Cunningham, and C. Hayes, "A biomechanical investigation of a single-limb squat: implications for lower extremity rehabilitation exercise," Journal of Athletic Training, vol. 43, no. 5, pp. 477-482, 2008.

[18] K. Hase, M. Sako, J. Ushiba, and N. Chino, "Motor strategies for initiating downward-oriented movements during standing in adults," Experimental Brain Research, vol. 158, no. 1, pp. 1827, 2004.

[19] V. C. Dionisio, G. L. Almeida, M. Duarte, and R. P. Hirata, "Kinematic, kinetic and EMG patterns during downward squatting," Journal of Electromyography and Kinesiology, vol. 18, no. 1, pp. 134-143, 2008.

[20] H. J. Hermens, B. Freriks, C. Disselhorst-Klug, and G. Rau, "Development of recommendations for SEMG sensors and sensor placement procedures," Journal of Electromyography and Kinesiology, vol. 10, no. 5, pp. 361-374, 2000.

[21] V. C. Dionisio, N. Marconi, I. D. Santos, and G. L. Lmeida, "Upward squatting in individuals with and without patellofemoral pain syndrome: abiomechanical study," Journal of Strength and Conditioning Research, vol. 25, no. 5, pp. 1437-1446, 2011.

[22] L. A. de Souza, M. D. B. Curtarelli, M. Mukherjee, and V. C. Dionisio, "The effect of the partially restricted sit-tostand task on biomechanical variables in subjects with and without Parkinson's disease," Journal of Electromyography and Kinesiology, vol. 21, no. 5, pp. 719-726, 2011.
[23] P. Konrad, The ABC of EMG: A Practical Introduction to Kinesiological Electromyography, Noraxon, Boston, Mass, USA, 2005.

[24] W. Mathiyakom, J. L. McNitt-Gray, P. Requejo, and K. Costa, "Modifying center of mass trajectory during sit-to-stand tasks redistributes the mechanical demand across the lower extremity joints," Clinical Biomechanics, vol. 20, no. 1, pp. 105-111, 2005.

[25] F. Alves, F. Oliveira, C. Junqueira, B. Azevedo, and V. C. Dionísio, "Analysis of electromyographic patterns during standard and declined squats," Revista Brasileira de Fisioterapia, vol. 13, no. 2, pp. 164-172, 2009.

[26] R. F. Escamilla, G. S. Fleisig, N. Zheng, S. W. Barrentine, K. E. Wilk, and J. R. Andrews, "Biomechanics of the knee during closed kinetic chain and open kinetic chain exercises," Medicine and Science in Sports and Exercise, vol. 30, no. 4, pp. 556-569, 1998.

[27] J. C. Ninos, J. J. Irrgang, R. Burdett, and J. R. Weiss, "Electromyographic analysis of the squat performed in self-selected lower extremity neutral rotation and $30 \circ$ of lower extremity turn-out from the self-selected neutral position," Journal of Orthopaedic and Sports Physical Therapy, vol. 25, no. 5, pp. 307-315, 1997. 


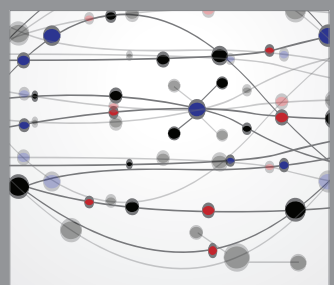

The Scientific World Journal
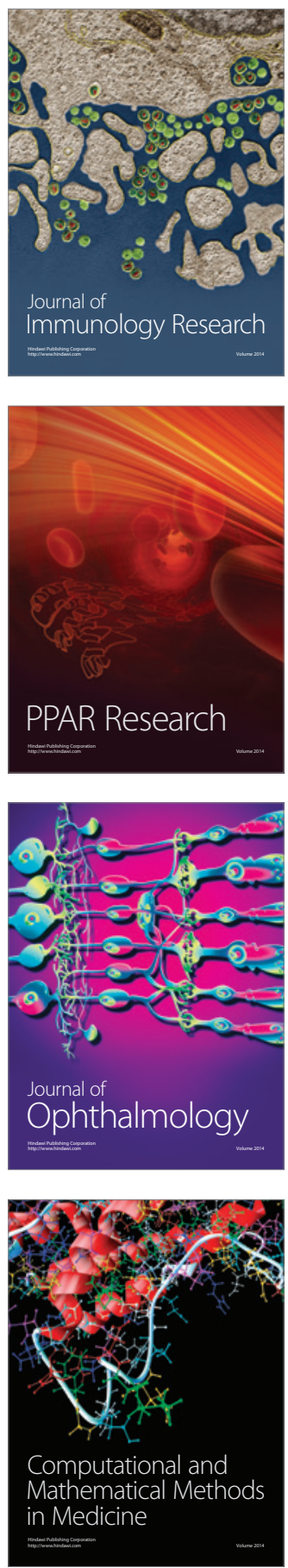

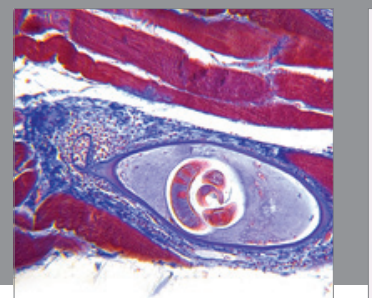

Gastroenterology

Research and Practice
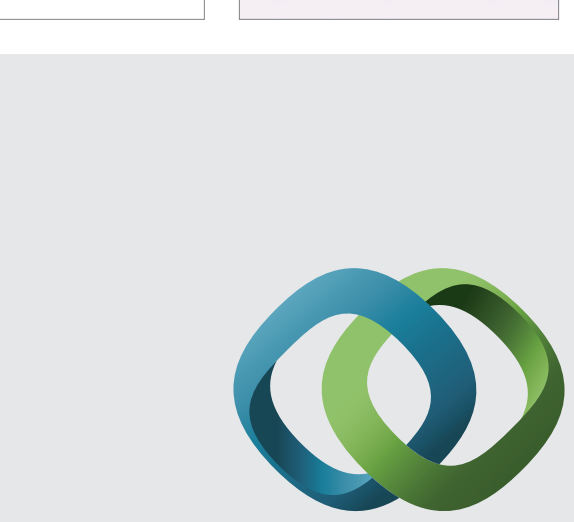

\section{Hindawi}

Submit your manuscripts at

http://www.hindawi.com
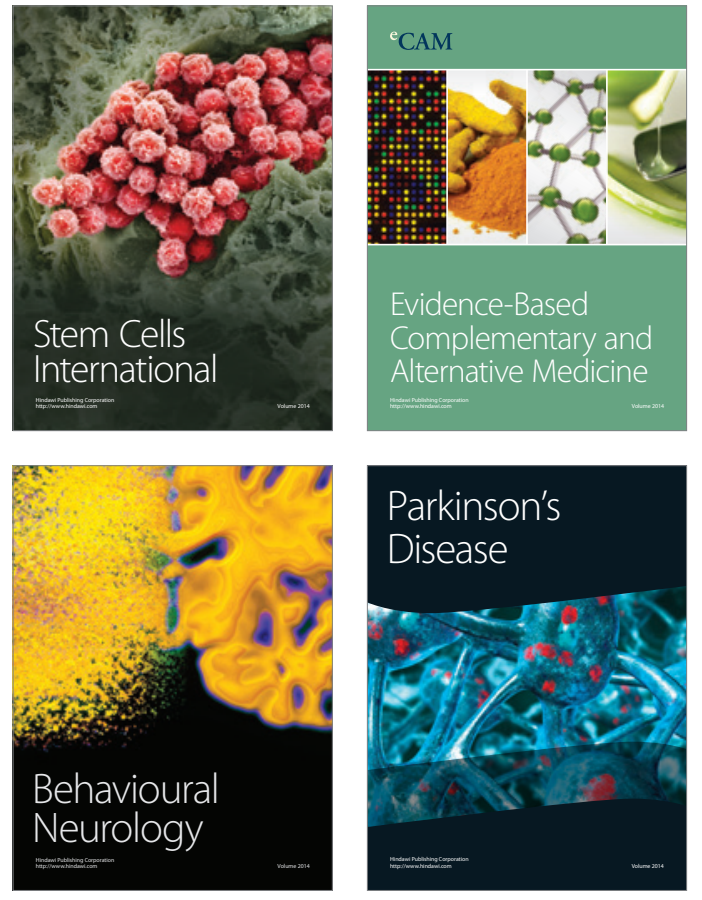
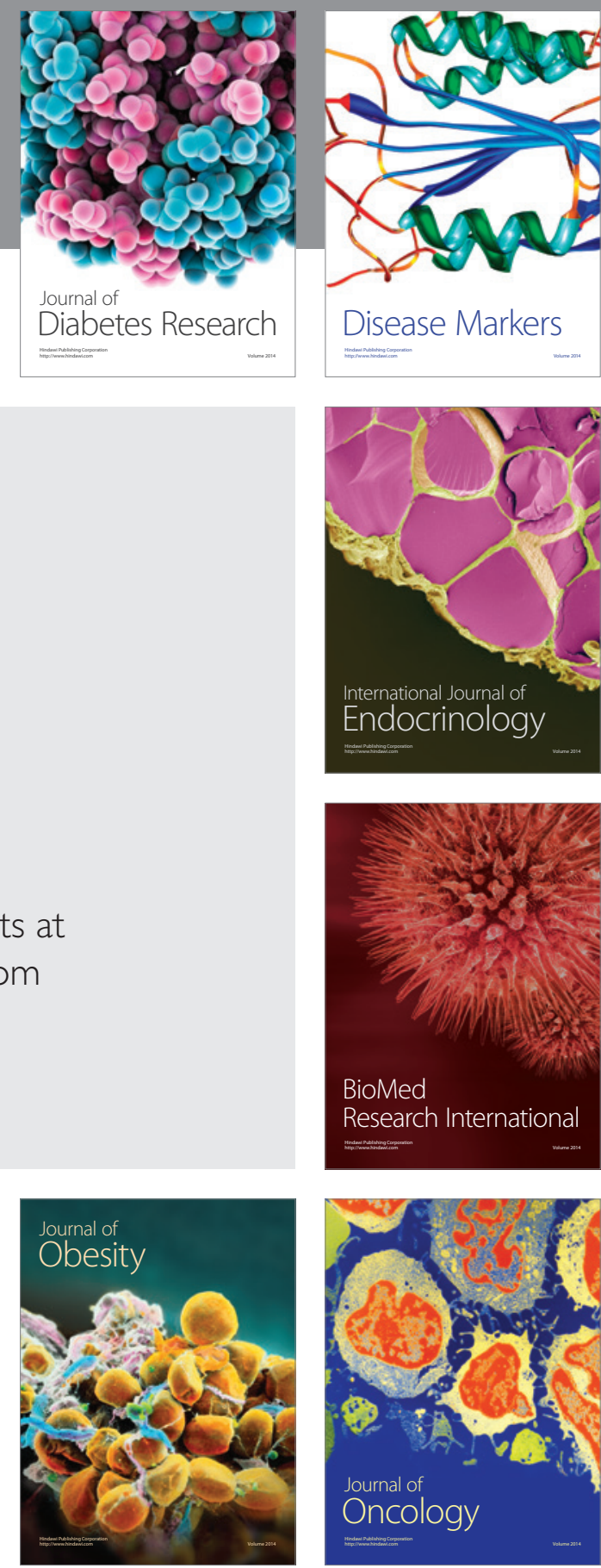

Disease Markers
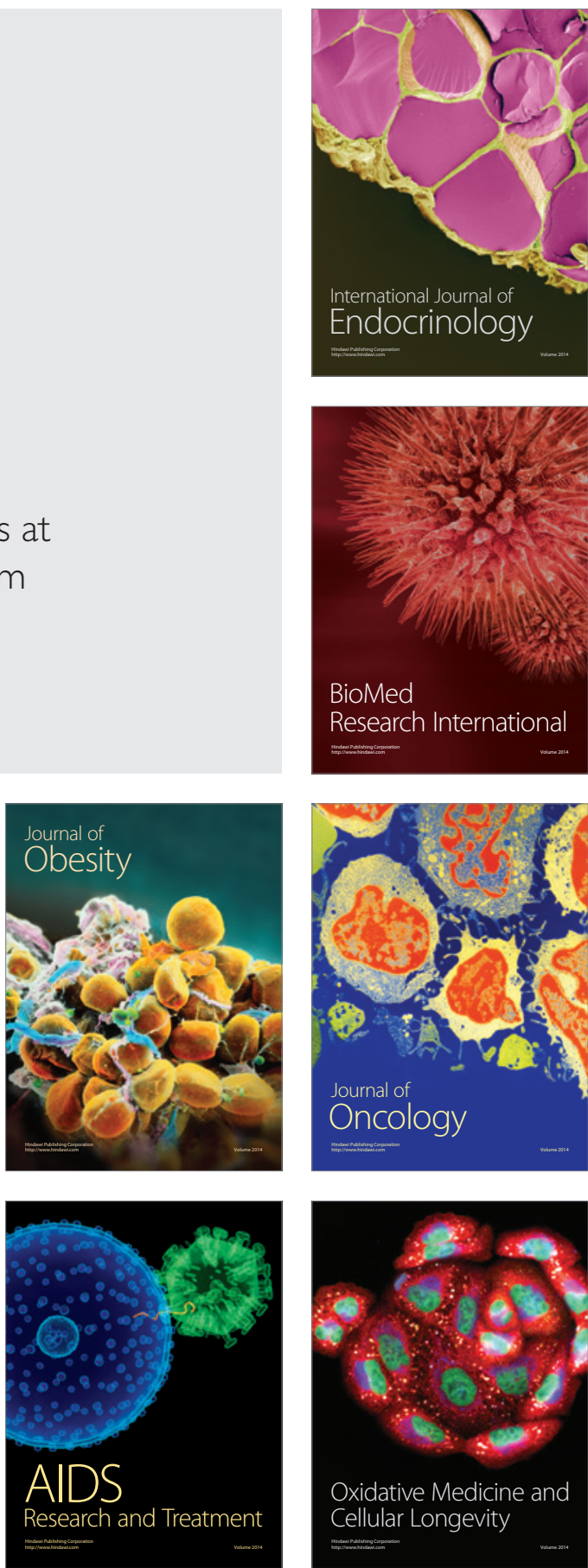